\title{
Efficient SLM Based Impulsive Noise Reduction in Powerline OFDM Communication Systems
}

\author{
Khaled M. Rabie and Emad Alsusa \\ Microwave and Communication Systems (MACS) Group, \\ School of Electrical and Electronic Engineering, \\ The University of Manchester, United Kingdom, M13 9PL, \\ Email: \{khaled.rabie, e.alsusa\}@manchester.ac.uk
}

\begin{abstract}
A very efficient method to mitigate impulsive noise (IN) over powerline channels is to precede the OFDM demodulator with a blanker to zero the incoming signal when it exceeds a certain threshold. Blanking the signal samples unaffected by IN exceeding this threshold, i.e. blanking errors, can cause severe performance degradation. For best performance, the optimal blanking threshold must be determined and this requires some prior and accurate knowledge about the characteristics of IN; this method is referred to as the unmodified method. In this paper, we propose an algorithm to enhance the capability of such methods by processing the OFDM signal at the transmitter to make the IN more easily identifiable at the receiver. This is done by simply deploying a peak to average power ratio (PAPR) reduction technique such as the selective mapping (SLM) scheme. A closed-form analytical expression for the probability of blanking error is derived and the problem of blanking threshold optimization is addressed under various IN environments. The results reveal that the proposed technique is able to minimize the probability of blanking error dramatically and can provide significant SNR improvement relative to the unmodified scheme. It will also be shown that when SLM is implemented with a large number of phase sequences, not only a considerable SNR enhancement is achieved but also, unlike the unmodified method, it becomes feasible to completely alleviate the need for any previous knowledge about the IN characteristics for optimal blanking.
\end{abstract}

Index Terms-Blanking, impulsive noise, OFDM, peak to average power ratio (PAPR), powerline communications (PLC), selective mapping (SLM), smart grid.

\section{INTRODUCTION}

$\mathbf{S}$ MART grid has been one of the most growing fields of research recently and can be attained via different technologies such as wireless, coaxial or powerline communications (PLC) [1]. PLC technology, however, is the most attractive for the realization of smart grid since it utilizes an existing infrastructure of wiring networks in addition to the ease of accessing electricity outlets in the home. This technology becomes even more appealing where radio signals suffer from high propagation loss, e.g. underground, underwater and building with metal walls [2]. On the other hand, PLC networks are classified as a harsh communication channel and in order to improve the reliability of such channels it is essential to overcome a number of challenges including the varying impedance of the wiring, high levels of frequency-dependent attenuation [3], [4] and the noise. Noise over powerlines is divided into two categories, [5], colored background noise (BN) and impulsive noise (IN) [6], [7]. The latter however is the most dominant factor that degrades the PLC signals and its power spectral density (PSD) always exceeds the
PSD of BN by at least $10-15 \mathrm{~dB}$ [8]. To study the impact of IN on PLC systems, few methods have been suggested to model the IN such as Middleton class-A noise model [6], [9]. This model has been the most widely accepted in the literature and therefore it will be adopted in this paper.

To improve the performance of the orthogonal frequency division multiplexing (OFDM) receivers over IN channels, several techniques have been introduced in the literature with varying degrees of complexity [10]-[12]. The simplest of which is to precede the conventional OFDM demodulator with a blanking device to zero the received signal with it exceeds a certain threshold [13], [14]. Blanking technique is widely used in practice because of its simplicity and ease of implementation. Theoretical performance analysis to find closed-form expressions for the signal-to-noise ratio (SNR) at the output of the blanker and optimization of blanking threshold first considered in [15], [16]. It is important stressing the fact that the main disadvantage of this method is that in order to optimally suppress the IN, the noise characteristics must be accurately known apriori in the form of signal-to-impulsive noise ratio (SINR) and the IN probability of occurrence. In this paper we refer to this technique as the unmodified method. Imperfect recognition of the signal samples corrupted by IN, also referred to as blanking errors, can lead to significant performance deterioration [17].

To our knowledge, all studies on IN mitigation are based on entirely countering IN at the receiver side and that no-one has considered preprocessing the OFDM signal at the transmitter side in order to enhance the IN cancellation process at the receiver. In this paper, it is proposed that the OFDM signal is processed at the transmitter in such a way to simplify and improve IN mitigation at the receiver. This could be done by simply applying a peak to average power ratio (PAPR) reduction technique. Techniques to reduce PAPR in OFDM systems include amplitude clipping, tone reservation (TR) and multiply signal representation techniques such as partial transmit sequence (PTS) and selective mapping (SLM) [18], [19]. In this paper, we exploit the SLM technique, which is well known for its robustness, and combine it with a blanking to conjointly minimize IN.

The contribution of this paper is twofold. The first contribution is to derive a closed-form expression for the probability of blanking error and demonstrate how it can be reduced considerably by the proposed technique. For more quantitative characterization, the corresponding output SNR is also considered. The second contribution resides in addressing the problem 


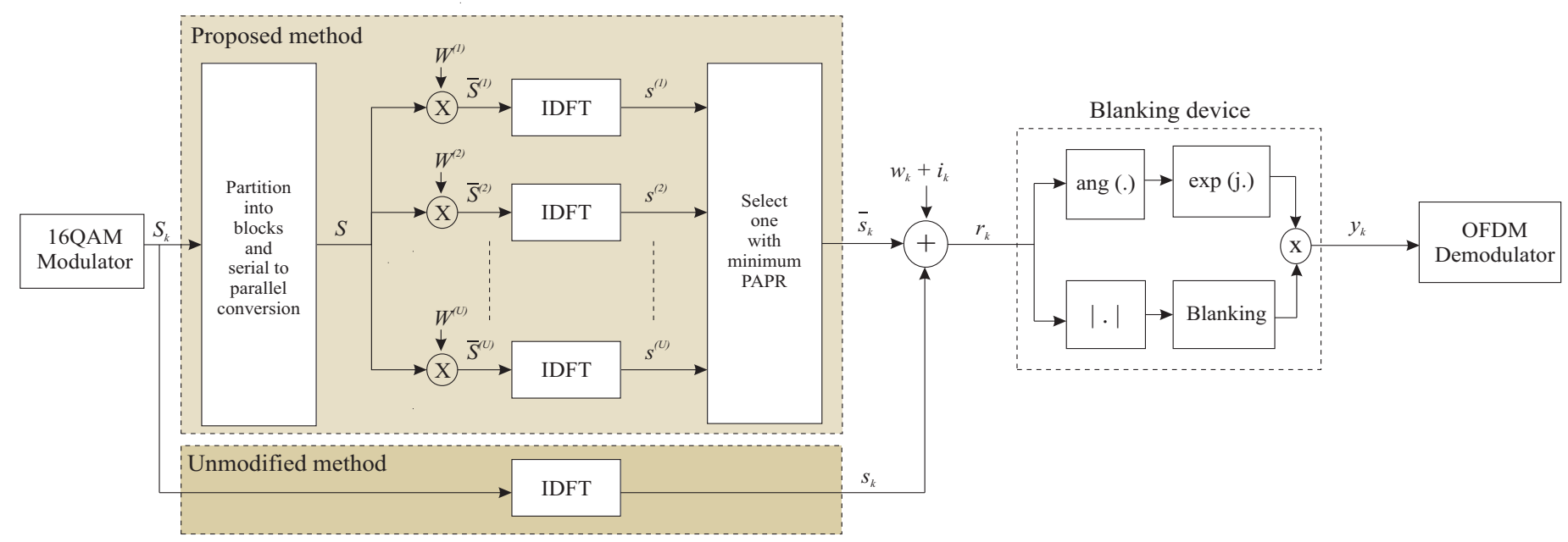

Fig. 1: Block diagram of OFDM system with SLM at the transmitter and blanking at the receiver

of blanking threshold optimization of the proposed technique for different phase sequences under various IN scenarios. The results reveal that the proposed can minimize the probability of blanking error significantly and is able to provide up to $2.75 \mathrm{~dB}$ SNR enhancement compared to the unmodified method. Most importantly, it will be shown that if SLM scheme is implemented with a sufficiently large number of phase sequences, the optimal blanking threshold (OBT) becomes independent of IN parameters. In contrast to the previous studies, this implies that the proposed can completely eliminate the need for any previous knowledge about the IN characteristics to blank it optimally.

The rest of the paper is organized as follows. In Section II, the system model is presented. In Section III, a closedform analytical expression for the probability of blanking error is derived for the proposed technique. Section IV outlines the simulation results including the output SNR performance and blanking threshold optimization. Finally, conclusions are drawn in Section V.

\section{System Model Overview}

The basic system model used in this study is shown in Fig. 1. This figure shows both the unmodified and the proposed systems. When the unmodified system (unmodified method block) is considered, the transmitted OFDM signal with $N$ subcarriers is defined as

$$
s(t)=\frac{1}{\sqrt{N}} \sum_{k=0}^{N-1} S_{k} e^{\frac{j 2 \pi k t}{T_{s}}}, \quad 0<t<T_{s}
$$

where $S_{k}$ represents the data sequence to be transmitted on the $k^{\text {th }}$ OFDM symbol and $T_{s}$ is the active symbol interval. The PAPR of the transmitted signal is given by

$$
\operatorname{PAPR}=\frac{\max |s(t)|^{2}}{\mathbb{E}\left[|s(t)|^{2}\right]}
$$

where $\mathbb{E}[$.$] is the expectation function. However, when the pro-$ posed method is considered, an SLM modulator is applied at the transmitter (proposed method block), see Fig. 1, which generates a set of $U$ different data blocks, representing the original data block, and then selects the one with the minimum PAPR for transmission. If $S=\left[S_{0}, S_{1}, \ldots, S_{N-1}\right]^{T}$ is the data stream after the serial-to-parallel converter, then each data block $S$ is multiplied by $U$ different phase sequence vectors $(W)$ each of length $N, W^{(u)}=\left[W_{0}^{(u)}, W_{1}^{(u)}, \ldots, W_{N-1}^{(u)}\right]^{T}, u=1,2, \ldots, U$. This multiplication yields $U$ modified data blocks as

$$
\bar{S}^{(u)}=\left[S_{0}^{(u)} W_{0}^{(u)}, W_{1}^{(u)} W_{1}^{(u)}, \ldots, S_{N-1}^{(u)} W_{N-1}^{(u)}\right]^{T}
$$

The modified blocks are then passed through the inverse discrete Fourier transform (IDFT) and the SLM-OFDM signal with $N$ sub-carriers is given by

$$
s^{(u)}(t)=\frac{1}{\sqrt{N}} \sum_{k=0}^{N-1} \bar{S}_{k}^{(u)} e^{\frac{j 2 \pi k t}{T_{s}}}, \quad 0<t<T_{s}
$$

Finally, the modified block with the minimum PAPR, $\bar{s}(t)$, will be selected for transmission and is found as

$$
\bar{s}(t)=\arg \min _{0 \leq u \leq U-1}\left\{\operatorname{PAPR}\left(s^{(u)}(t)\right)\right\}
$$

The OFDM or SLM-OFDM signal is then transmitted over the PLC channel where the noise is added to it. In this paper we adopt the Bernoulli-Gaussian noise model [20], which takes the following form

$$
n_{k}=w_{k}+i_{k}, \quad k=0,1,2, \ldots, N-1
$$

where

$$
i_{k}=b_{k} g_{k}, \quad k=0,1,2, \ldots, N-1
$$

$n_{k}$ is the total noise component, $w_{k}$ is the additive white Gaussian noise (AWGN), $i_{k}$ is the IN, $g_{k}$ is complex white Gaussian noise with mean zero and $b_{k}$ is the Bernoulli process with probability mass function

$$
\operatorname{Pr}\left(b_{k}\right)=\left\{\begin{array}{ll}
p, & b_{k}=1 \\
0, & b_{k}=0
\end{array} \quad k=0,1, \ldots, N-1\right.
$$


$p$ denotes the probability occurrence of IN. The probability density function (PDF) of the total noise can be expressed as

$$
P_{n_{k}}\left(n_{k}\right)=(1-p) \mathcal{G}\left(n_{k}, 0, \sigma_{w}^{2}\right)+p \mathcal{G}\left(n_{k}, 0, \sigma_{w}^{2}+\sigma_{i}^{2}\right)
$$

where $\mathcal{G}($.$) is the Gaussian PDF given by (10) whereas \sigma_{w}^{2}$ and $\sigma_{i}^{2}$ are the AWGN and IN variances, respectively.

$$
\mathcal{G}\left(x, \mu, \sigma_{x}^{2}\right)=\frac{1}{\sqrt{2 \pi \sigma_{x}^{2}}} e^{-\frac{(x-\mu)^{2}}{2 \sigma_{x}^{2}}}
$$

The AWGN and IN variances define the input SNR and SINR as in (11) and (12), respectively, where $\sigma_{s}^{2}$ is the transmitted signal power.

$$
\begin{aligned}
& \mathrm{SNR}=10 \log _{10}\left(\frac{\sigma_{s}^{2}}{\sigma_{w}^{2}}\right) \\
& \mathrm{SINR}=10 \log _{10}\left(\frac{\sigma_{s}^{2}}{\sigma_{i}^{2}}\right)
\end{aligned}
$$

Under perfect synchronization condition, the received signal is given by

$$
r_{k}=\left\{\begin{array}{ll}
s_{k} / \overline{s_{k}}+w_{k}, & \mathcal{H}_{0} \\
s_{k} / \overline{s_{k}}+w_{k}+i_{k}, & \mathcal{H}_{1}
\end{array} \quad k=0,1, \ldots, N-1\right.
$$

$s_{k}=s\left(k T_{s} / N\right)$ and $\overline{s_{k}}=\bar{s}\left(k T_{s} / N\right)$. The null hypothesis $\mathcal{H}_{0}$ implies the absence of IN, $P\left(\mathcal{H}_{0}\right)=(1-p)$; whereas the alternative hypothesis $\mathcal{H}_{1}$ implies the presence of IN, $P\left(\mathcal{H}_{1}\right)=$ $p$. Also, $s_{k}, w_{k}$ and $i_{k}$ are assumed to be mutually independent. At the receiver side, $r_{k}$ is fed to the blanking device which works as follows

$$
y_{k}=\left\{\begin{array}{ll}
r_{k}, & \left|r_{k}\right| \leq T \\
0, & \left|r_{k}\right|>T
\end{array} \quad k=0,1, \ldots, N-1\right.
$$

where $T$ is the blanking threshold. $r_{k}$ and $y_{k}$ are the input and output of the blanker, respectively. The threshold $T$ should be carefully selected to maximize the system performance. In [15], a theoretical expression for the OBT $\left(T_{o p t}\right)$ of the unmodified method was derived as a function of IN parameters (15) as well as the output SNR which is given as

$$
\mathrm{SNR}_{\text {unmod }}=\frac{2}{\mathbb{E}\left[A_{n}^{2}\right]}
$$

where $\mathbb{E}\left[A_{n}^{2}\right]$ is defined as in (17). These expressions will be used to provide a comparative analysis to show the superiority of the proposed technique and also to verify the accuracy of our simulation model.

\section{Probability of Blanking Error}

One way of assessing the proposed technique is the probability of blanking error $\left(P_{b_{e}}\right)$ which is defined as the probability that the amplitude of the received sample, $A_{r}=\left|r_{k}\right|$, exceeds the blanking threshold when it is unaffected by IN and it is expressed as

$$
P_{b_{e}}=P\left(B, \mathcal{H}_{0}\right)=P\left(A_{r}>T \mid \mathcal{H}_{0}\right) P\left(\mathcal{H}_{0}\right)
$$

$$
=\left[1-F_{A_{r}}\left(T \mid \mathcal{H}_{0}\right)\right] P\left(\mathcal{H}_{0}\right)
$$

$F_{A_{r}}\left(T \mid \mathcal{H}_{0}\right)$ is the conditional cumulative distribution function (CDF) and $B$ is the even of blanking the received signal exceeding $T$. In the absence of IN, the amplitude of the received signal has Rayleigh distribution with parameter $\sigma^{2}=\sigma_{s}^{2}+\sigma_{w}^{2}$. Therefore, for the unmodified method $P_{b_{e}}$ is given as

$$
P_{b_{e}}^{\text {unmod }}=e^{-\left(\frac{T^{2}}{2\left(\sigma_{s}^{2}+\sigma_{w}^{2}\right)}\right)}(1-p)
$$

In the case of SLM-OFDM system, the PDF of the transmitted signal as a function of $N$ and $U$ is derived in [21] and is reproduced for convenience in (20). Some numerical results of this expression are presented in Fig. 2 in the absence of IN (substituting $\sigma^{2}=\sigma_{s}^{2}+\sigma_{w}^{2}$ ) for several values of $U$. Simulation results are also provided and it can be seen that both results are in good agreement. It can be observed that at probability of $10^{-3}$, the received signal (transmitted signal + Gaussian noise) exceeding a certain threshold is reduced by one unit. The conditional CDF of the SLM-OFDM system is found as

$$
\begin{aligned}
F_{A_{r}}^{S L M} & \left(T \mid \mathcal{H}_{0}\right)=\left.\int_{-\infty}^{T} f^{S L M}(x) \mathrm{d} x\right|_{\sigma^{2}=\sigma_{s}^{2}+\sigma_{w}^{2}} \\
= & \left(1-\left[1-\left(1-e^{-\frac{T^{2}}{2\left(\sigma_{s}^{2}+\sigma_{w}^{2}\right)}}\right)^{N}\right]^{U}\right)^{\frac{1}{N}}
\end{aligned}
$$

Using the definition of $P_{b_{e}}$ in (18) we can write the probability of blanking error of the SLM-OFDM system $\left(P_{b_{e}}^{S L M}\right)$ as in (23). Some numerical results obtained from (23) are shown in Fig. 3 along with simulation results for an OFDM system with $N=128$ and input $\mathrm{SNR}=30 \mathrm{~dB}$ and various values of $U$. It is noticeable that simulation results closely match the analytical ones. In general, there are two trends that can be observed from this figure. First it is clear that as $U$ increases, the probability of blanking error is minimized for a given value of blanking threshold. The other observation is the fact that for the same value of $U$ the enhancement in the probability increases rapidly when the blanking threshold is increased. For instance, when $U=8$ and at blanking threshold of 2.75 , the probability is reduced by about 0.5 order of magnitude compared to the unmodified system $(U=1)$ whereas for blanking threshold of 3.25 the probability is minimized by about 3 orders of magnitude. This implies that the system performance will improve for higher values of $U$ as presented in the next section.

\section{Simulation Results}

In this section, extensive computer simulations are conducted to examine the performance of the proposed technique in terms of the output SNR. In addition, the OBT that maximizes the output SNR is investigated. The simulation parameters are: OFDM system with $N=64$ sub-carriers, 16QAM modulation, OFDM signal power is normalized to unity $\sigma_{s}^{2}=(1 / 2) \mathbb{E}\left[\left|s_{k}\right|^{2}\right]=1$, $\sigma_{w}^{2}=(1 / 2) \mathbb{E}\left[\left|w_{k}\right|^{2}\right]$ and $\sigma_{i}^{2}=(1 / 2) \mathbb{E}\left[\left|i_{k}\right|^{2}\right]$.

The output SNR is found by (24). In our investigations we set $\mathrm{SNR}=40 \mathrm{~dB}, \mathrm{SINR}=-10 \mathrm{~dB}$ and $p=0.01$. 


$$
T_{\text {opt }}=\sqrt{\frac{2\left(1+\sigma_{w}^{2}\right)\left(1+\sigma_{w}^{2}+\sigma_{i}^{2}\right)}{\sigma_{i}^{2}} \ln \left(\left[\frac{1+\sigma_{w}^{2}+\sigma_{i}^{2}}{1+\sigma_{w}^{2}}\right]^{2} \frac{\left(1-\sigma_{w}^{2}\right)}{\left(1-\sigma_{w}^{2}-\sigma_{i}^{2}\right)} \frac{(p-1)}{p}\right)}
$$

$$
\begin{aligned}
E\left[A_{n}^{2}\right]=2(1-p)\left[\sigma_{w}^{2}\left(1-\sigma_{w}^{2}\right)\right. & \left(\frac{T^{2}}{2\left(1+\sigma_{w}^{2}\right)}+1\right) e^{\left.-\frac{T^{2}}{2\left(1+\sigma_{w}^{2}\right)}\right]} \\
+ & 2 p\left[\left(\sigma_{w}^{2}+\sigma_{i}^{2}\right)+\left(1-\sigma_{w}^{2}-\sigma_{i}^{2}\right)\left(\frac{T^{2}}{2\left(1+\sigma_{w}^{2}+\sigma_{i}^{2}\right)}+1\right) e^{-\frac{T^{2}}{2\left(1+\sigma_{w}^{2}+\sigma_{i}^{2}\right)}}\right]
\end{aligned}
$$

$$
f^{S L M}(x)=U f(x)\left(\left(1-e^{-\frac{x^{2}}{2 \sigma^{2}}}\right)^{N}\right)^{U-1}\left(1-\left(1-\left(1-e^{-\frac{x^{2}}{2 \sigma^{2}}}\right)^{N}\right)^{U}\right)^{\frac{1}{N}}
$$

$$
P_{b_{e}}^{S L M}=\left(1-F_{A_{r}}^{S L M}\left(T \mid \mathcal{H}_{0}\right)\right) P\left(\mathcal{H}_{0}\right)=\left[1-\left(1-\left(1-\left(1-e^{\left(-\frac{T^{2}}{2\left(\sigma_{s}^{2}+\sigma_{w}^{2}\right)}\right)}\right)^{N}\right)^{U}\right)^{\frac{1}{N}}\right](1-p)
$$

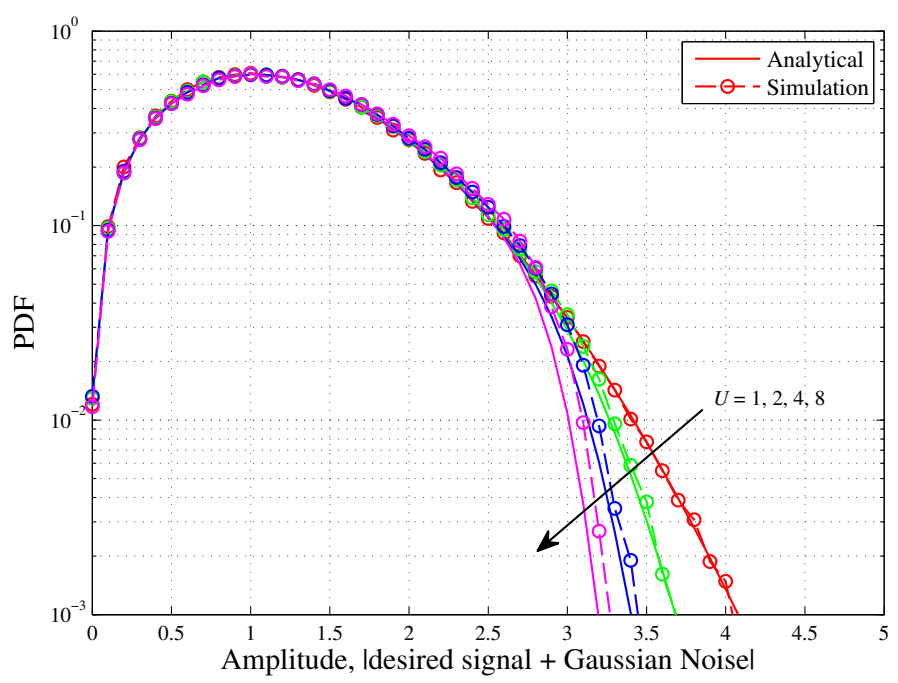

Fig. 2: PDF of 16QAM-SLM-OFDM signals with different values of $U$ and $\mathrm{SNR}=30 \mathrm{~dB}, N=128$

$$
\operatorname{SNR}_{S L M}^{U}=\frac{\mathbb{E}\left[\left|\overline{s_{k}}\right|^{2}\right]}{\mathbb{E}\left[\left|y_{k}-\overline{s_{k}}\right|^{2}\right]}
$$

\section{A. The Output SNR versus Blanking Threshold}

The output SNR is plotted as a function of the blanking threshold in Fig. 4 for an SLM-OFDM system with different values of $U$. It is seen that when $U=1$ (unmodified method), the analytical results obtained from (16) and the simulation ones are matching. This conveys that our simulation model can be reliably used to assess the performance of the proposed technique. As anticipated, one can see that as $U$ increases the output SNR improves and it can also be observed that for each

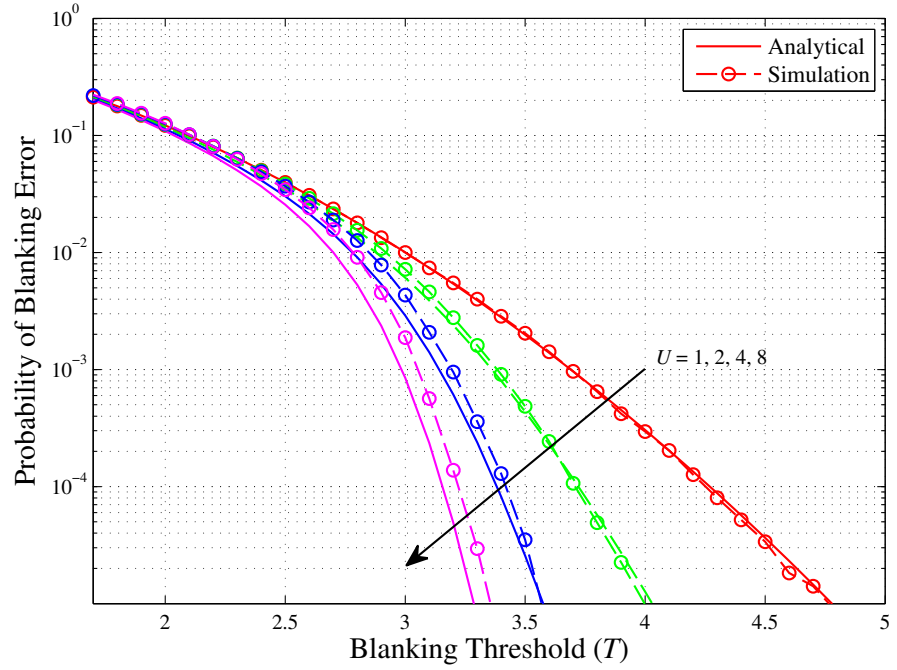

Fig. 3: Probability of blanking error for 16QAM-SLM-OFDM signals with different values of $U$ and SNR $=30 \mathrm{~dB}, N=128$

value of $U$ there exists an OBT at which the output SNR is maximized. Besides, as $U$ increases the OBT deceases and higher SNR is achieved. The optimization of blanking threshold of the SLM-OFDM system is discussed next.

\section{B. The Blanking Threshold Optimization}

In this subsection extensive simulations have been carried out to optimize the blanking threshold for different values of $U$ which is found by satisfying the following argument

$$
T_{o p t}^{U}=\arg \max _{0 \leq T<\infty}\left\{\operatorname{SNR}_{S L M}^{U}(T, p, \mathrm{SINR}, \mathrm{SNR})\right\}
$$

Fig. 5 depicts the OBT versus SINR for $U=\{1,2,4,8,16$, $32,64\}$. The analytical OBT results of the unmodified method 


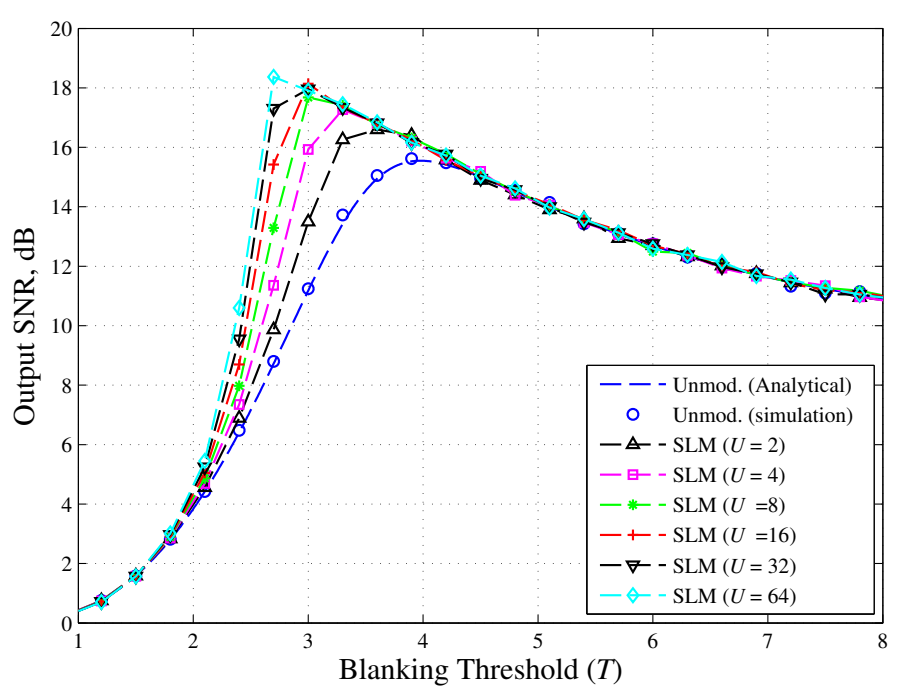

Fig. 4: The output SNR versus blanking threshold for various values of $U$, $\mathrm{SNR}=40 \mathrm{~dB}$, SINR $=-10 \mathrm{~dB}$ and $p=0.01$.

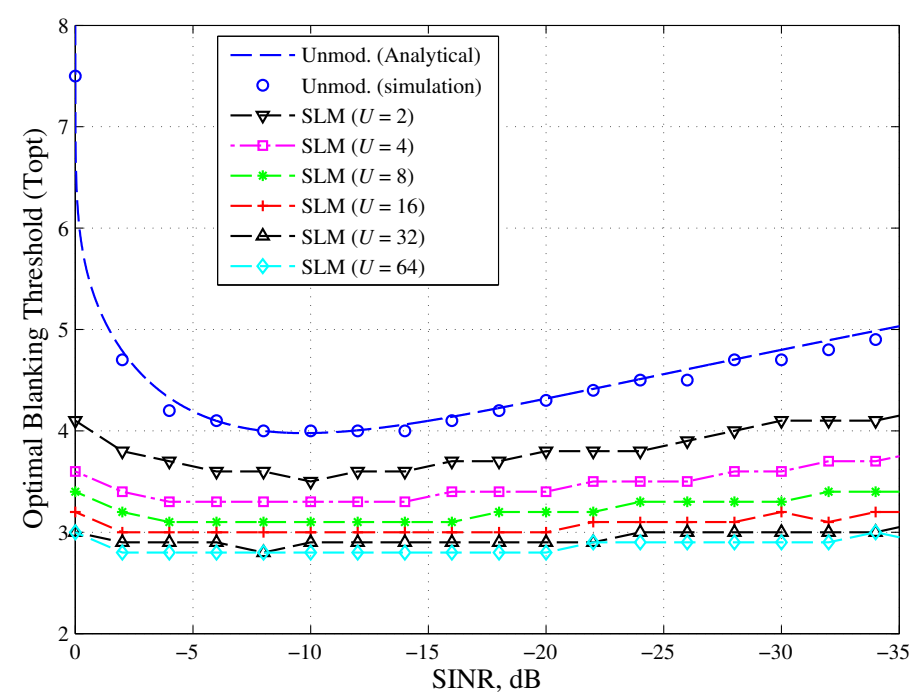

Fig. 5: Optimal blanking threshold versus SINR for various values of $U$, $\mathrm{SNR}=40 \mathrm{~dB}$ and $p=0.01$.

are obtained from (15). One common observation one can clearly see is that when $U$ is increased, the OBT becomes smaller. This can be intuitively justified as follows: when $U$ increases, the useful signal energy will be contained within lower level and hence smaller blanking threshold will allow more efficient blanking of the IN. Furthermore, it is important to point out that for large values of $U(U \geq 16)$ the OBT levels off, i.e the OBT becomes independent of SINR. This implies that if we deploy an SLM-OFDM system with a sufficiently large number of phase sequences, it will become possible to optimally blank the IN independently of the noise characteristics.

\section{Maximum Achievable Output SNR}

The maximum achievable SNR at the output of the blanker corresponding to the OBT found in the previous subsection is presented in Fig. 6 versus SINR for different values $U$. It is clear

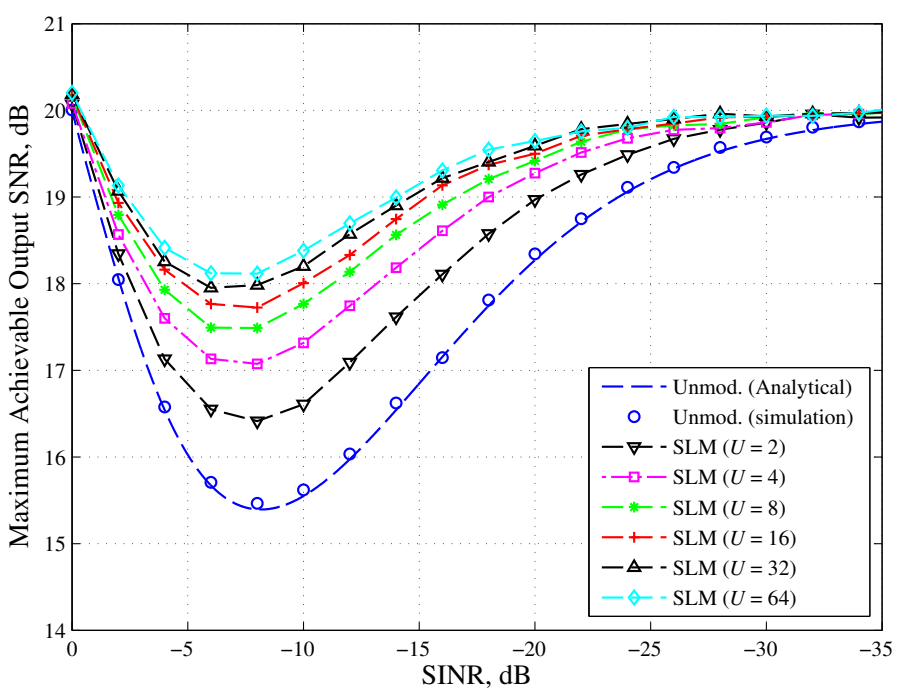

Fig. 6: Maximum achievable output SNR versus SINR for different values of $U, \mathrm{SNR}=40 \mathrm{~dB}$ and $p=0.01$.

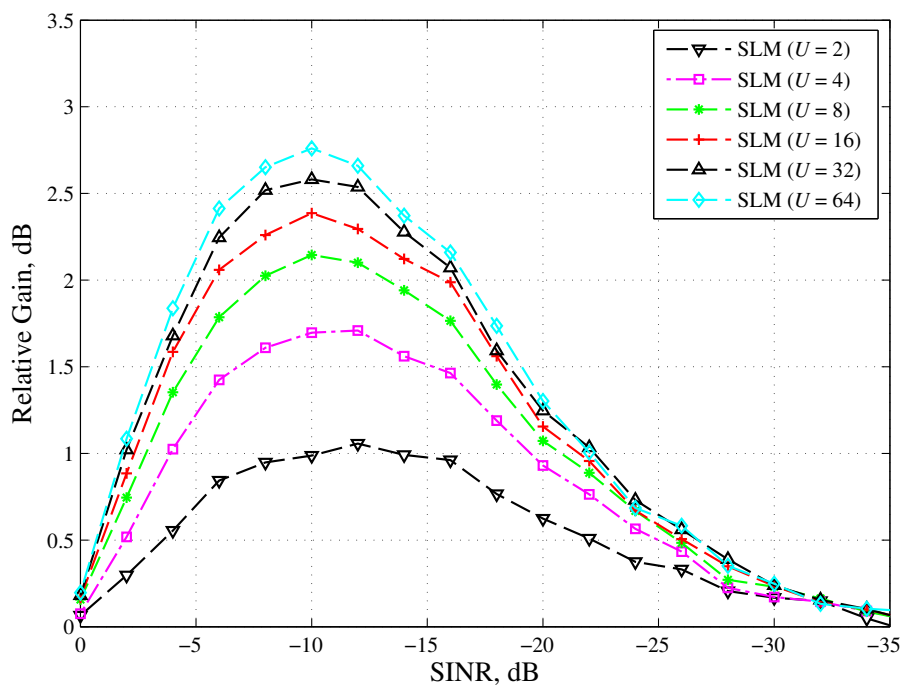

Fig. 7: Relative gain versus SINR for different values of $U, \mathrm{SNR}=40 \mathrm{~dB}$ and $p=0.01$.

that the proposed technique always outperforms the unmodified method and this enhancement is proportional to $U$. To highlight this phenomena, we have plotted the relative gain $\left(G_{R}\right)$, given by (26), versus SINR in Fig. 7.

$$
G_{R}=10 \log _{10}\left(\frac{\operatorname{SNR}_{S L M}\left(T=T_{o p t}^{U}\right)}{\operatorname{SNR}_{\text {unmod }}\left(T=T_{o p t}^{(U=1)}\right)}\right)
$$

It is evident that the largest improvement is reached in the intermediate SINR region $(-5 \mathrm{~dB} \rightarrow-15 \mathrm{~dB})$ where a gain of up to $2.75 \mathrm{~dB}$ is achieved in the output SNR over the unmodified method when $U=64$. It is also worthwhile mentioning the fact that ever for small number of phase sequences $(U=2)$ the proposed can still provide a gain of about $1 \mathrm{~dB}$. Furthermore, it is clear that this gain becomes negligible in the low SINR region $($ SINR $\rightarrow-\infty)$. This is due to the fact that in this region IN amplitude is extremely high and can easily be identified and 
hence can be completely eliminated even with the unmodified method.

\section{CONCLusion}

In this paper we have proposed a new method to improve the conventional blanking technique to reduce the effect of IN by employing a PAPR reduction scheme, namely SLM. The results clearly demonstrate the robustness and superiority of the proposed method in the form of minimized probability of blanking error and an increase in the output SNR which can be as high as $2.75 \mathrm{~dB}$. It was also found that when SLM scheme is applied with a sufficiently large number of phase sequences $(U \geq 64)$, the IN can be blanked optimally without the need to prior knowledge about it characteristics. However, this enhancement is attained at the expense of some computational complexity at the transmitter. Although we have considered only blanking in this paper, the proposed technique can be further examined with OFDM systems utilizing other nonlinear preprocessors such as clipping and hybrid.

\section{REFERENCES}

[1] B. Adebisi, A. Treytl, A. Haidine, A. Portnoy, R. Shan, D. Lund, H. Pille, and B. Honary, "IP-centric high rate narrowband PLC for smart grid applications," IEEE Commun. Mag., vol. 49, no. 12, pp. 46 -54, Dec. 2011.

[2] X. Cheng, R. Cao, and L. Yang, "Relay-aided amplify-and-forward powerline communications," IEEE Trans. Smart Grid, vol. PP, no. 99, pp. 1 $-8,2013$.

[3] D. Anastasiadou and T. Antonakopoulos, "Multipath characterization of indoor power-line networks," IEEE Trans. Power Del., vol. 20, no. 1, pp. 90-99, Jan. 2005.

[4] M.-Y. Zhai, "Transmission characteristics of low-voltage distribution networks in china under the smart grids environment," IEEE Trans. Power Del., vol. 26, no. 1, pp. $173-180$, jan. 2011.

[5] H. Philipps, "Development of a statistical model for powerline communication channels," International Symposium on Power Line Communications (ISPLC), pp. 153-160, Apr. 2000.

[6] D. Middleton, "Canonical and quasi-canonical probability models of class A interference," IEEE Trans. Electromagn. Compat., vol. EMC-25, pp. 76-106, May 1983.
[7] _ _ "Non-gaussian noise models in signal processing for telecommunications: new methods an results for class A and class B noise models," IEEE Trans. Inform. Theory, vol. 45, no. 4, pp. 1129 -1149, May 1999.

[8] M. Zimmermann and K. Dostert, "Analysis and modeling of impulsive noise in broad-band powerline communications," IEEE Trans. Electromagn. Compat., vol. 44, pp. 249-258, Feb. 2002.

[9] D. Middleton, "Statistical-physical models of electromagnetic interference," IEEE Trans. Electromagn. Compat., vol. EMC-19, pp. 106-127, Aug. 1977.

[10] E. Del Re, R. Fantacci, S. Morosi, and R. Seravalle, "Comparison of cdma and ofdm techniques for downstream power-line communications on low voltage grid," IEEE Trans. Power Del., vol. 18, no. 4, pp. 1104 - 1109, Oct. 2003.

[11] J. Haring and A. J. H. Vinck, "Iterative decoding of codes over complex numbers for impulsive noise channels," IEEE Trans. Inf. Theory, vol. 49, no. 5, pp. 1251-1260, May 2003.

[12] S. V. Zhidkov, "Impulsive noise suppression in OFDM-based communication systems," IEEE Trans. Consum. Electron., vol. 49, no. 4, pp. 944-948, Nov. 2003.

[13] O. P. H. et al., "Detection and removal of clipping in multicarrier receivers," European patent application EP1043874, Oct. 2011.

[14] N. P. Cowley, A. Payne, and M. Dawkins, "COFDM tuner with impulse noise reduction," Eur. Patent Application EP1180851, Feb. 2002.

[15] S. V. Zhidkov, "On the analysis of ofdm receiver with blanking nonlinearity in impulsive noise channels," Intelligent Signal Processing and Cоттиnication Systems, 2004. ISPACS 2004. Proceedings of 2004 International Symposium on, pp. 492-496, Nov. 2004.

[16] - "Performance analysis and optimization of OFDM receiver with blanking nonlinearity in impulsive noise environment," IEEE Trans. Veh. Technol., vol. 55, no. 1, pp. 234-242, Jan. 2006.

[17] E. Alsusa and K. Rabie, "Dynamic peak based threshold estimation method for mitigating impulsive noise in powerline communication systems," IEEE Trans. Power Del., 2013, Accepted.

[18] R. W. Baaauml, R. F. H. Fisher, and J. B. Huber, "Reducing the peakto-average power ratio of multicarrier modulation by selected mapping," Elect. Lett., vol. 32, no. 22, pp. 2056-57, Oct. 1996.

[19] H. Breiling, S. H. Muuuller-Weinfurtner, and J. B. Huber, "SLM peakpower reduction without explicit side information," IEEE Commun. Lett., vol. 5, no. 6, pp. 239-41, Jun. 2001.

[20] M. Ghosh, "Analysis of the effect of impulse noise on multicarrier and single carrier QAM systems," IEEE Trans. Commun., vol. 44, no. 2, pp. 145-147, Feb. 1996.

[21] H. Yoo, F. Guilloud, and R. Pyndiah, "Amplitude PDF analysis of OFDM signal using probabilistic PAPR reduction method," EURASIP Journal on Wireless Communications and Networking, vol. 2011, 2011. 